\title{
ESSENTIAL SPECTRA OF ELLIPTIC PARTIAL DIFFERENTIAL EQUATIONS ${ }^{1}$
}

\author{
BY MARTIN SCHECHTER
}

\author{
Communicated by Jürgen Moser, February 15, 1967
}

Let $A$ be a closed, densely defined operator in a Banach space $X$. There are several definitions of the "essential" spectrum of $A$ (cf. [1], [2]). According to Wolf [3], [4] it is the complement in the complex plane of the $\Phi$-set of $A$. The $\Phi$-set $\Phi_{A}$ of $A$ is the set of points $\lambda$ for which

(a) $\alpha(A-\lambda)$, the dimension of the null space of $A-\lambda$, is finite

(b) $R(A-\lambda)$, the range of $A-\lambda$, is closed

(c) $\beta(A-\lambda)$, the codimension of $\mathrm{R}(A-\lambda)$, is finite.

We denote the essential spectrum according to this definition by $\sigma_{e w}(A)$. The set $\sigma_{e m}(A)$, as defined in [1], [2] is obtained by adding to $\sigma_{e v}(A)$ those points $\lambda$ for which $\alpha(A-\lambda) \neq \beta(A-\lambda)$. It is the largest subset of $\sigma(A)$ which remains invariant under compact perturbations. Finally, to obtain the set $\sigma_{e b}(A)$, which is the essential spectrum according to Browder [5], we add to $\sigma_{e m}(A)$ those points of $\sigma(A)$ which are not isolated.

Interest in the sets $\sigma_{e w}(A), \sigma_{e m}(A), \sigma_{e b}(A)$ is centered about the fact that they remain invariant under certain perturbations of $A$. In particular one has

TheOREM 1. Let $A$ and $B$ be closed densely defined operators in $X$. If $\lambda_{0} \in \rho(A) \cap \rho(B)$ and $\left(A-\lambda_{0}\right)^{-1}-\left(B-\lambda_{0}\right)^{-1}$ is a compact operator in $X$, then

$$
\sigma_{e w}(A)=\sigma_{e w}(B)
$$

and

$$
\sigma_{e m}(A)=\sigma_{e m}(B) .
$$

Moreover, if the complement $\mathbf{C} \sigma_{e m}(A)$ of $\sigma_{e m}(A)$ is connected, then

$$
\sigma_{e b}(A)=\sigma_{e b}(B) .
$$

This theorem was proved in [2] under the additional assumption that $D(B) \supseteq D(A)$. For selfadjoint operators the basic idea was employed by Birman [6], Wolf [4] and Rejto [7].

1 Research supported in part by National Science Foundation Grant GP-5676 and in part by a NSF Senior Postdoctoral Fellowship. 
We shall apply Theorem 1 to the situation of an elliptic operator perturbed by a potential. Let

$$
A(x, D)=\sum_{|\mu|,|\nu| \leq r} D^{\mu} a_{\mu \nu}(x) D^{\nu}
$$

be a uniformly strongly elliptic operator of order $2 r$ defined in the whole of $n$-dimensional Euclidean space $E^{n}$. Here $\mu=\left(\mu_{1}, \cdots, \mu_{n}\right)$, $\nu=\left(\nu_{1}, \cdots, \nu_{n}\right)$ are multi-indices of nonnegative integers, $|\mu|$ $=\mu_{1}+\cdots+\mu_{n}, D^{\mu}=(-i)^{|\mu| \partial|\mu|} / \partial x_{1}^{\mu_{1}} \cdots \partial x_{n}^{\mu_{n}}$. The coefficients $a_{\mu \nu}(x)$ are to have bounded derivatives of all orders $\leqq \max (|\mu|,|\nu|)$ in $E^{n}$ and for $|\mu|=|\nu|=r$ the $a_{\mu \nu}(x)$ are to be uniformly continuous in $E^{n}$. By uniform strong ellipticity we mean that there is a constant $C_{0}>0$ such that

$$
\operatorname{Re} \sum_{|\mu|=|\nu|=r} a_{\mu \nu}(x) \xi^{\mu} \xi^{\nu} \geqq C_{0}|\xi|^{2 r}
$$

for real vectors $\xi=\left(\xi_{1}, \cdots, \xi_{n}\right)$ and all $x \in E^{n}$, where $|\xi|^{2}=\xi_{1}^{2}+\cdots$ $+\xi_{n}^{2}, \xi^{\mu}=\xi_{1}^{\mu_{1}} \cdots \xi_{n}^{\mu_{n}}$.

Let $A_{0}$ be the operator $A(x, D)$ acting on the set $C_{0}^{\infty}$ of infinitely differentiable functions with compact supports. We shall see that there is an extension of $A_{0}$ containing a half plane in its resolvent set.

Let $A$ be a densely defined linear operator in a Hilbert space $H$. According to Kato [8] it is called regularly accretive if there is a bilinear form $a(u, v)$ such that

(1) $D(a) \supseteq D(A)$

(2) $\operatorname{Re} a$ is closed, and there is a constant $\gamma>0$ such that

$$
\operatorname{Re} a(u, u) \geqq \gamma|\operatorname{Im} a(u, u)| \quad \text { for } u \in D(a)
$$

(3) For $u \in D(a)$ and $f \in H$ one has

$$
a(u, v)=(f, v) \text { for all } v \in D(a)
$$

if and only if $u \in D(A)$ and $A u=f$.

We call $a(u, v)$ a bilinear form if it is linear in $u$ and conjugate linear in $v$. We write $a(u)$ in place of $a(u, u)$ and call $a(u, v)$ closed if $u_{n} \in D(a), u_{n} \rightarrow u$ in $H$ and $a\left(u_{n}-u_{m}\right) \rightarrow 0$ imply that $u \in D(a)$ and $a\left(u_{n}\right) \rightarrow a(u)$. It is called preclosed if $u_{n} \in D(a), u_{n} \rightarrow 0, a\left(u_{n}-u_{m}\right) \rightarrow 0$ imply $a\left(u_{n}\right) \rightarrow 0$. It is easily seen that a preclosed nonnegative symmetric form has a closure (cf. [9]). The real and imaginary parts of a bilinear form are defined as

$\operatorname{Re} a(u, v)=\frac{1}{2}[a(u, v)+\overline{a(v, u)}] ; \quad \operatorname{Im} a(u, v)=\frac{1}{2 i}[a(u, v)-\overline{a(v, u)}]$. 
One can show easily that the bilinear form corresponding to a regularly accretive operator is unique. Let $A$ be a regularly accretive extension of an operator $A_{0}$. We shall call $A$ minimal if for any regularly accretive extension $A^{\prime}$ of $A_{0}$ we have

(i) $D(a) \subseteq D\left(a^{\prime}\right)$,

(ii) $a^{\prime}(u, v)=a(u, v), u, v \in D(a)$,

where $a$ and $a^{\prime}$ are the bilinear forms corresponding to $A$ and $A^{\prime}$, respectively.

The following theorem is basic in our study and answers a question raised by Kato [8].

Theorem 2. Let $A_{0}$ be a densely defined linear operator in $H$. Then a necessary and sufficient condition that $A_{0}$ have a regularly accretive extension is that

$$
\operatorname{Re}\left(A_{0} u, u\right) \geqq \gamma\left|\operatorname{Im}\left(A_{0} u, u\right)\right| u \in D\left(A_{0}\right)
$$

holds for some constant $\gamma>0$. Moreover, there is a minimal extension which satisfies ( 7$)$ with the same constant $\gamma$.

Returning to our operator $A(x, D)$ we wish to determine conditions on a function $q(x)$ so that $A_{0}+q+\lambda$ has a regularly accretive extension. We formulate our conditions in terms of the following expressions (compare [10]). We define

$$
\begin{aligned}
M_{\alpha, p}(q) & =\sup _{\chi} \int_{|x-y|<1}|q(y)|^{p}|x-y|^{\alpha} d y \quad-n<\alpha<0 \\
& =\sup _{\chi} \int_{|x-y|<1}|q(y)|^{p}\left(1+\log \frac{1}{|x-y|}\right) d y \quad \alpha=0 \\
& =\sup _{\chi} \int_{|x-y|<1}|q(y)|^{p} d y \quad \alpha>0
\end{aligned}
$$

We let $M_{\alpha, p}$ be the set of functions $q$ for which $M_{\alpha, p}(q)<\infty$.

For any function $h(x)$ we set $h^{+}(x)=\max [0, h(x)]$ and $h^{-}(x)$ $=\min [0, h(x)]$. Employing Theorem 2, we have

Theorem 3. Assume that $\operatorname{Im} q \in M_{2 r-n, 1}$ and that $(\operatorname{Re} q)^{-} \in M_{\alpha, 1}$ for some $\alpha$ satisfying $-n<\alpha<2 r-n$. Then there is a $\lambda_{0}>0$ such that $A_{0}$ $+q+\lambda$ has a regularly accretive extension for each $\lambda>\lambda_{0}$.

We now apply Theorem 1 to obtain

TheOREM 4. Let $q$ satisfy the hypotheses of Theorem 3 and, in addition, assume that $(\operatorname{Re} q)^{+} \in M_{\beta, 1}, \beta<4 r-n$, and 


$$
\int_{|x-y|<1}|q(y)| d y \rightarrow 0 \text { as }|x| \rightarrow \infty .
$$

Let $A$ and $B$ be the minimal regularly accretive extensions of $A_{0}+\lambda$ and $A_{0}+q+\lambda$, respectively, $\lambda>\lambda_{0}$. Then (1) and (2) hold. If $\mathrm{C}_{\sigma_{e m}}(A)$ is connected, then (3) holds.

CoRollary 5. If Im $q \in M_{2 r-n, 1}, \operatorname{Re} q \in M_{\alpha, 1}$ and (8) holds, then the conclusions of Theorems 3 and 4 hold.

Next we assume that there are constants $a_{\mu \nu}$ such that for each $\mu, \nu,|\mu|,|\nu| \leqq r$,

$$
\int_{|x-y|<1}\left|a_{\mu \nu}(y)-a_{\mu \nu}\right| d y \rightarrow 0 \quad \text { as }|x| \rightarrow \infty .
$$

We set

$$
A(\infty, D)=\sum_{|\mu|,|\nu| \leq r} D^{\mu} a_{\mu \nu} D^{\nu}
$$

and let $A_{\infty}$ be the minimal operator of $A(\infty, D)$, i.e., the closure in $L^{2}$ of $A(\infty, D)$ defined on $C_{0}^{\infty}$. The spectrum of $A_{\infty}+\lambda$ is easily computed via Fourier transforms and consists of the set $R_{\lambda}$ of those complex $\eta$ for which there is a real vector $\xi$ satisfying $\sum_{|\mu|,|\nu| \text { s } r} a_{\mu \nu} \xi \xi^{\eta}+\lambda$ $=\eta$. Moreover, if $\eta$ is in $\sigma\left(A_{\infty}\right)$, then the range of $A_{\infty}-\eta$ is not closed in $L^{2}$. Hence we have

$$
\sigma_{e w}\left(A_{\infty}\right)=\sigma_{e m}\left(A_{\infty}\right)=\sigma_{e b}\left(A_{\infty}\right)=\sigma\left(A_{\infty}\right)=R_{0} .
$$

We can now state

TheOREM 6. If (9) holds as well as the assumptions of Theorems 3 and 4, we have

$$
\sigma_{e w}(B)=\sigma_{e m}(B)=R_{\lambda},
$$

where $B$ is the minimal regularly accretive extension of $A_{0}+q+\lambda$. If $\mathrm{C} R_{\lambda}$ is connected, we have

$$
\sigma_{e b}(B)=R_{\lambda}
$$

as well.

In the next two theorems we consider the perturbation of $A(x, D)$ by another operator of the same form

$$
C(x, D)=\sum_{|\mu|,|\nu| \leq r} D^{\mu} C_{\mu \nu}(x) D^{\nu} .
$$


We assume that $D^{\mu}\left(C_{\mu \nu} u\right) \in L^{2}$ for $u \in C_{0}^{\infty}$ and let $C_{0}$ denote the operator $C(x, D)$ with domain $C_{0}^{\infty}$. We let $M_{-n, 2}$ denote the set of essentially bounded functions and take $\alpha$ to satisfy $2 r-n-2<\alpha<2 r-n$. We make two sets of assumptions

H1. (a) $\left(\operatorname{Re} C_{\mu \mu}\right)-\in M_{\alpha-2|\mu|, 1}$ for $|\mu| \neq r$, $\left(\operatorname{Re} C_{\mu \mu}\right)^{-}=0$ for $|\mu|=r$

(b) For $\mu \neq \nu$

$$
C_{\mu \nu}(x)+\bar{C}_{\nu \mu}(x)=g_{1 \mu \nu}(x) h_{1 \mu \nu}(x)
$$

where

$$
\begin{aligned}
& g_{1 \mu \nu}(x) \in M_{2 r-2|\mu|-n, 2}, \quad h_{1 \mu \nu}(x) \in M_{\alpha-2|\nu|, 2} \quad|\nu| \neq r \\
& g_{1 \mu \nu}(x) \in M_{\alpha-2|\mu|, 2}, \quad h_{1 \mu \nu}(x) \in M_{-n, 2}, \quad|\mu| \neq r, \quad|\nu|=r \\
& g_{1 \mu \nu}(x) \equiv 0 \quad|\mu|=|\nu|=r \\
& \text { (c) } C_{\mu \nu}(x)-\bar{C}_{\nu \mu}(x)=g_{2 \mu \nu}(x) h_{2 \mu \nu}(x)
\end{aligned}
$$

where

$$
g_{2 \mu \nu}(x) \in M_{2 r-2|\mu|-n, 2}, \quad h_{2 \mu \nu}(x) \in M_{2 r-2|p|-n, 2}
$$

H2. (a) $\left(\operatorname{Re} C_{\mu \mu}\right)+\in M_{\beta-2|\mu|, 1}, \quad \beta<4 r-n$

(b) $\int_{|x-y|<1}\left|C_{\mu \nu}(y)\right| d y \rightarrow 0$ as $|x| \rightarrow \infty$.

TheOREM 7. Under assumptions $\mathrm{H} 1$ there is $a \lambda_{0}>0$ such that $A_{0}+C_{0}+\lambda$ has a regularly accretive extension for each $\lambda>\lambda_{0}$.

TheORem 8. Under assumptions $\mathrm{H} 1$ and $\mathrm{H} 2$, we have

$$
\sigma_{e w}(E)=\sigma_{e w}(A), \quad \sigma_{e m}(E)=\sigma_{e m}(A),
$$

where $E$ is the minimal regularly accretive extension of $A_{0}+C_{0}+\lambda$. If $\boldsymbol{C} \sigma_{e m}(A)$ is connected, then $\sigma_{e b}(E)=\sigma_{e b}(A)$. If (9) holds, then

$$
\sigma_{e w}(E)=\sigma_{e m}(E)=R_{\lambda} .
$$

\section{BIBLIOGRAPHY}

1. Martin Schechter, Invariance of the essential spectrum, Bull. Amer. Math. Soc. 71 (1965), 365-367.

2. - On the essential spectrum of an arbitrary operator. I, J. Math. Anal. Appl. 13 (1966), 205-215.

3. František Wolf, On the essential spectrum of partial differential boundary problems, Comm. Pure Appl. Math. 12 (1959), 211-228.

4. - On the invariance of the essential spectrum under a change of boundary conditions of partial differential boundary operators, Indag. Math. 21 (1959), 142-315.

5. F. E. Browder, On the spectral theory of elliptic differential operators. I, Math. Ann. 142 (1961), 22-130. 
6. M. S. Birman, On the spectrum of singular boundary problems, Mat. Sb. 55(2) 97 (1961), 125-174.

7. P. A. Rejto, On the essential spectrum of the hydrogen energy operator, Pacific J. Math. 19 (1966), 109-140.

8. Tosio Kato, Fractional powers of dissipative operators, J. Math. Soc. Japan 13 (1961), 246-274.

9. - Quadratic forms in hilbert spaces and asymptotic perturbation series, Technical Report No. 7, University of California, 1955.

10. Erik Balslev, The essential spectrum of elliptic differential operators in $L^{p}\left(R_{n}\right)$, Trans. Amer. Math. Soc. 116 (1965), 193-217.

11. Nachman Aronszajn and K. T. Smith, Theory of Bessel potentials. I, Technical Report No. 22, University of Kansas, 1959.

12. A. P. Calderón, Lebesgue spaces of differentiable functions and distributions, Proceeding of Symposia in Pure Mathematics Vol. 4, American Mathematical Society, Providence, R.I., 1961, 33-49.

13. Lars Hörmander, Linear partial differential operators, Springer, Berlin, 1963.

14. P. D. Lax and A. N. Milgram, Parabolic equations, Annals of Math. Studies, No. 33, Princeton, 1954, 167-190.

15. Erik Balslev, The essential spectrum of self-adjoint elleptic differential operators in $L^{2}\left(R_{n}\right)$, Math. Scand. (to appear).

Belfer Graduate School of Science, Yeshiva University

\title{
REPRESENTATIONS OF UNIFORMLY HYPERFINITE ALGEBRAS AND THEIR ASSOCIATED VON NEUMANNN RINGS
}

\author{
BY ROBERT T. POWERS
}

Communicated by W. Browder, February 16, 1967

Introduction. In this note we summarize the main results of a paper, Representations of uniformly hyperfinite algebras and their associated von Neumann rings, which will be published elsewhere.

A uniformly hyperfinite (UHF) algebra of class $\left\{n_{i}\right\}$ is a $C^{*}$ algebra, $\mathfrak{A}$, which contains an increasing sequence of factors, $M_{1} \subset M_{2} \subset \cdots \subset \mathfrak{A}$, of types, $\left(\mathrm{I}_{n_{1}}\right),\left(\mathrm{I}_{n_{2}}\right), \cdots$, such that $\mathfrak{A}$ is the norm closure of $\bigcup_{i=1}^{\infty} M_{i}$. It is always assumed that the integers, $n_{i} \rightarrow \infty$ as $i \rightarrow \infty$. UHF algebras have been defined and studied by Glimm [2].

If $\Pi$ is a ${ }^{*}$-representation of a UHF algebra, $\mathfrak{A}$, on a Hilbert space, then the von Neumann ring, $R=\{\Pi(\mathfrak{C})\}^{\prime \prime}$, generated by the representation algebra, $\Pi(\mathfrak{R})$, has the property that $R$ is the strong closure of an increasing sequence of type $\left(\mathrm{I}_{n}\right)$ factors. Von Neumann rings with this property will be called hyperfinite rings. It is clear that every 